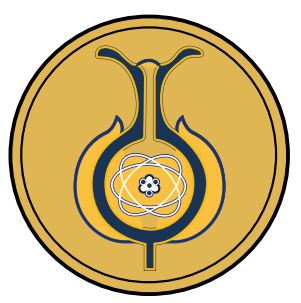

\title{
Redes sociales en áreas silvestres protegidas de Costa Rica
}

\author{
Social networks in wildlife protected areas of Costa Rica \\ Redes sociais em áreas naturais protegidas da Costa Rica
}

\section{Melissa Marín Cabrera'}

Received: Dec/18//2020 • Accepted: Jun/16/2021 • Published: Jan/31/2022

\section{Resumen (18)}

Este artículo describe de forma comparativa las redes de participación social en tresáreas silvestres protegidas del Pacífico Central de Costa Rica. Estas áreas representan distintas categorías de manejo, es decir, distintas posibilidades de aprovechamiento de los recursos naturales. La metodología utilizó un enfoque mixto para ampliar las formas de entender los procesos socioambientales en estas áreas. Las herramientas de análisis incluyeron: la teoría de grafos, el análisis de redes sociales y los mapas de calor con variables atributivas e indicadores globales y posicionales de las redes. Los elementos de estudio consideraron 40 grupos organizados en los años 2009-2010, y 40 en los años 2019-2020, todos vinculados al desarrollo local y al medio ambiente. Las unidades de análisis fueron las interacciones que se dan entre ellos. Los resultados muestran que a mayor centralidad en las redes y menor el poder de los grupos locales y multisectoriales, mayor es la desvinculación de los temas que atañen a la sostenibilidad de las áreas silvestres protegidas y mayor es la vulnerabilidad ante cambios estructurales y externos. Entre las conclusiones destaca que, desde la dimensión estructural, las redes de participación sí están influenciadas por las categorías de manejo debido a que condicionan el acceso que se tenga a los servicios ecosistémicos que estas protegen. Desde la dimensión funcional, la capacidad de las redes para transformar esos servicios ecosistémicos en oportunidades de desarrollo es independiente de las categorías de manejo, pero sí está relacionada con otros aspectos sociales.

Palabras clave: áreas silvestres protegidas; redes sociales; grupos organizados; participación social.

\section{Abstract (10)}

This article compares social participation networks in three wildlife protected areas in the Central Pacific of Costa Rica. These areas represent different management categories, which means different approaches to the use of natural resources. A mixed approach methodology was used to have a wider understanding of the socio-environmental processes in these areas. Analyses used included graph theory, social network analysis, and heat maps with attribute variables and global and positional network indicators. The elements of analysis included 40 civil society groups related to local development and environment conservation organized between 2009-2010 and 40 between 2019-2020. The analysis units were the interactions among the groups. Results show that the more

Melissa Marín Cabrera, \ mmarincr@gmail.com, (DD : https://orcid.org/0000-0001-8696-932X

1 Programa de Doctorado en Ciencias Naturales para el Desarrollo, (Universidad Nacional de Costa Rica, Instituto Tecnológico de Costa Rica y Universidad Estatal a Distancia), San José, Costa Rica. 
centralized the social networks and the less power of local and multisectoral groups, the greater the dissociation of the topics related to sustainability of wildlife protected areas and the more vulnerable to structural and external changes. It is concluded that, from the structural dimension, participation networks are influenced by the management categories, since they condition the access to their ecosystem services. From the functional dimension, the capability of participation networks to transform ecosystem services into development opportunities is irrespective of the management categories, although it is related to other social aspects.

Keywords: wildlife protected areas; social networks; organized groups; organizational participation.

\section{Resumo (10)}

Este artigo descreve de forma comparativa as redes de participação social em três áreas naturais protegidas do Pacífico Central da Costa Rica. Essas áreas representam diferentes categorias de gestão, ou seja, diferentes possibilidades de uso dos recursos naturais. A metodologia utilizou uma abordagem mista para ampliar as formas de compreensão dos processos socioambientais nessas áreas. As ferramentas de análise incluíram: teoria dos grafos, análise de redes sociais e mapas de calor com variáveis atributivas e indicadores globais e posicionais das redes. Os elementos do estudo consideraram 40 grupos organizados nos anos 2009-2010, e 40 nos anos 2019-2020, todos vinculados ao desenvolvimento local e ao meio ambiente. As unidades de análise foram as interações que ocorrem entre elas. Os resultados mostram que quanto maior a centralidade das redes e menor o poder dos grupos locais e multissetoriais, maior a desvinculação de questões relacionadas à sustentabilidade das áreas naturais protegidas e maior a vulnerabilidade às mudanças estruturais e externas. Entre as conclusões destaca-se que, a partir da dimensão estrutural, as redes de participação são influenciadas pelas categorias de gestão, pois condicionam o acesso aos serviços ecossistêmicos que protegem. A partir da dimensão funcional, a capacidade das redes de transformar esses serviços ecossistêmicos em oportunidades de desenvolvimento é independente das categorias de gestão, mas está relacionada a outros aspectos sociais.

Palavras-chave: áreas naturais protegidas; redes sociais; grupos organizados; participaç̧ão social.

\section{Introducción}

Las redes sociales son espacios ideales para describir los patrones de colaboración e interacción social. Sus características estructurales permiten comprender los procesos sociales, es decir, cómo las personas definen el poder, las formas de distribución de recursos, cómo se dan los flujos de información, cómo inciden en áreas geográficas específicas y cómo todo lo anterior puede relacionarse con la funcionalidad de una red para atender a las necesidades humanas fundamentales, según la definición de Neef, Elizalde y Hopenhayn (1986).

Además, en las redes sociales se crean las capacidades tanto individuales como colectivas, o sea, es donde se sustenta el capital social. Coleman (1990) define el capital social como el conjunto de recursos disponibles con que el individuo cuenta a raíz de su participación en redes sociales. Aquí convergen una dimensión estructural (Sabatini, 2009) y una dimensión funcional (Häuberer, 2011). 
Lo anterior es especialmente importante para el análisis de la funcionalidad de los sistemas sociales (en términos de efectividad) para conservar y hacer uso sostenible de los bienes y servicios ecosistémicos en áreas silvestres protegidas.

Según Mardones (2017), en estas áreas confluye una multiplicidad de actores en diferentes dimensiones espaciales y temporales, con intereses muchas veces divergentes, pero que requieren de una mayor integración para hacer converger los propósitos de la conservación y el desarrollo.

Una de las formas de analizar esta integración es a partir de las relaciones que los actores construyen de forma individual, pero también aquellas que se dan entre grupos de actores. Sobre esto último, y en relación con las áreas silvestres protegidas, Maya, Florido del Corral y Sáenz. (2017) mencionan que el conocimiento ecológico local y la construcción de una visión integrada del ecosistema están influenciados también por las relaciones que vinculan grupos de actores con diferentes recursos e información.

A pesar de que el estudio de las redes sociales es uno de los campos de investigación de mayor desarrollo dentro de las ciencias sociales (Ávila, 2012), y que, según Palacio (2017), la acción ambiental es uno de sus campos emergentes (p.73), en Costa Rica el uso de investigación cuantitativa para explicar estructuralmente los procesos de conservación y de gestión de los recursos naturales es todavía muy limitado. A pesar de lo anterior, y aunque no es específico sobre áreas silvestres protegidas, sí se destacan las investigaciones de Samper (2004) sobre redes de experimentadores campesinos; $y$ el Programa Estado de la Nación en Desarrollo Humano Sostenible (2014) sobre redes en tecnología e investigaciones científicas.
En Costa Rica, el 25,5\% del territorio terrestre e insular y el $2,6 \%$ de la zona económica exclusiva se encuentran protegidos mediante alguna de las 9 categorías de manejo (SINAC, 2020). Estas fueron adaptadas a partir de las 7 categorías recomendadas por la Unión Internacional para la Conservación de la Naturaleza (UICN) (Dudley, 2008) y tienen el objetivo de facilitar la planificación de territorios claves para la biodiversidad en función de sus necesidades de protección y su vulnerabilidad frente alteraciones antrópicas, climáticas, entre otras. Esto implica formas distintas de gestión y limitaciones en el uso de los recursos naturales, siendo la categoría I la más restrictiva y la VII, la menos restrictiva.

Las áreas silvestres protegidas, además de resguardar la biodiversidad y otros valores ambientales necesarios para la resiliencia de los sistemas naturales, también han sido generadores de oportunidades directas o indirectas para el desarrollo.

Por ejemplo, a nivel mundial, se estimó que para el 2019 el turismo de vida silvestre generó 346.000 millones de USD de forma directa e indirecta y 21,8 millones de puestos de trabajo (WTTC, 2019). En Costa Rica, esta actividad provee el $13,2 \%$ del total de empleo (Benavides, 2020), e incluye aquel generado a través del ecoturismo en áreas silvestres protegidas con importantes beneficios (pero también dependencia) para las economías locales. Tal es el caso de las comunidades alrededor del Parque Nacional Tortuguero, el Parque Nacional Manuel Antonio, o la Reserva Privada Bosque Nuboso de Monteverde.

El recurso hídrico que se conserva en las áreas silvestres protegidas, particularmente los ecosistemas de humedal, también es fuente importante de desarrollo si se aprovecha de forma sostenible. Los 
sistemas agrícolas también se ven beneficiados debido a la presencia y diversidad de polinizadores, que mejora su productividad, da un valor agregado y brinda mayor resiliencia ante las fluctuaciones del mercado o los cambios del clima.

Las posibilidades para el desarrollo que brindan las áreas silvestres protegidas las ha convertido en zonas socialmente dinámicas. El creciente interés por conservar sus recursos, por aprovecharlos o por participar en su gestión ha derivado en relaciones de colaboración y de conflicto, con influencias positivas y negativas en la biodiversidad y en el desarrollo.

En este sentido, la Ley de Biodiversidad de Costa Rica N. 7788 establece los Comités Locales de Áreas de Conservación (COLAC) y la Ley 2726, las Asociaciones Administradoras de los Sistemas de Acueductos y Alcantarillados Comunales (ASADAS) como estrategias de gobernanza participativas para aumentar la colaboración alrededor de las áreas silvestres protegidas y reducir los conflictos por el uso irracional de sus recursos.

Sin embargo, el interés por los servicios ecosistémicos no siempre se ha traducido en la existencia o funcionalidad de estrategias formales de gobernanza, pero sí en el surgimiento de redes informales para restringir, controlar, aumentar o diversificar el uso de los recursos naturales. Este es el caso de las tres áreas de estudio que se describen más adelante.

Atendiendo a lo mencionado por Bodin y Crona (2009), que indican que las redes sociales pueden ser más importantes que la existencia de instituciones formales para la efectiva aplicación y cumplimiento de la normativa ambiental (p. 366), y por Marín et al. 2012 (citado en Mardones, 2017, p. 63) en relación a que la estructura social de una comunidad puede ser un elemento facilitador u obstaculizador para la conservación de la biodiversidad y la integración con estrategias de desarrollo local, este artículo describe y analiza las estructuras de los mecanismos informales de participación (representado por redes de grupos organizados) alrededor de tres áreas silvestres protegidas y su posible incidencia en el desarro1lo. Se comparan categorías de manejo para identificar la influencia de distintos niveles de acceso a los servicios ecosistémicos en la construcción de las redes sociales.

La hipótesis de investigación se centra en que la contribución de las áreas silvestres protegidas al desarrollo de las comunidades aledañas no está directamente vinculada a los tipos de categorías de manejo de los recursos naturales, sino a las características de las redes de participación en la gestión ambiental alrededor de ellas y su capacidad de potenciar y distribuir los bienes y servicios que estas áreas generan.

Además, se busca comprobar la utilidad de la teoría de grafos y el análisis de redes sociales como herramientas cuantitativas para ampliar las formas de entender los procesos socioambientales.

\section{Metodología}

La metodología se basó en un enfoque mixto a través del análisis de redes sociales (Wasserman y Faust, 1994, Diani y McAdam, 2003 y Hanneman, 2000) e información cualitativa. Esta se representó con la teoría de grafos y mapas de calor. La información se recopiló durante los años 2009, 2010, 2019 y 2020. 


\section{Área de estudio}

El espacio geográfico de análisis fueron las siguientes tres áreas silvestres:

1) Reserva Biológica Cerro Vueltas (categoría I de UICN). Creada en el año 1995 mediante el Decreto Ejecutivo No 24439-MIRENEM. Posee una extensión de 793,27 hectáreas (ha) (SINAC, 2015) con importantes valores ecológicos de las zonas altas de montaña (Kappelle y Brown, 2001 y Gentry, 1982), un reducto de páramo subalpino poco alterado, además de 9 sistemas hídricos que son afluentes de los principales ríos de la zona (SINAC, 2015). En esta categoría de manejo no es permitido ningún uso, salvo investigaciones científicas con el seguimiento por parte de las autoridades ambientales.

2) Parque Nacional Los Quetzales (categoría II de UICN). Creado por el Decreto Ejecutivo No 32981 en 2006 con una extensión de 4.117,09 ha (SINAC, 2017). Surgió para detener la deforestación (principalmente de robles, Quercus costaricensis y $Q$. copeyensis) en la Reserva Forestal Los Santos. En esta categoría solo se permiten actividades de bajo impacto, como turismo ecológico, investigación y educación ambiental.

3) Reserva Forestal Los Santos (categoría VI de UICN). Creada mediante el Decreto Ejecutivo No 5389-A en el año 1975, cuenta actualmente con 56.389 ha. Según la legislación de Costa Rica, en esta categoría el uso de sus recursos naturales es más amplio con el debido control por parte del Estado. Se permite la presencia de poblados.
Geográficamente estas tres áreas limitan unas con otras (Figura 1), por lo que, en términos ecológicos, actúan como zonas de amortiguamiento entre sí. Socialmente esto también es importante, puesto que permite analizar las redes de participación considerando patrones de desarrollo históricos y culturales similares.

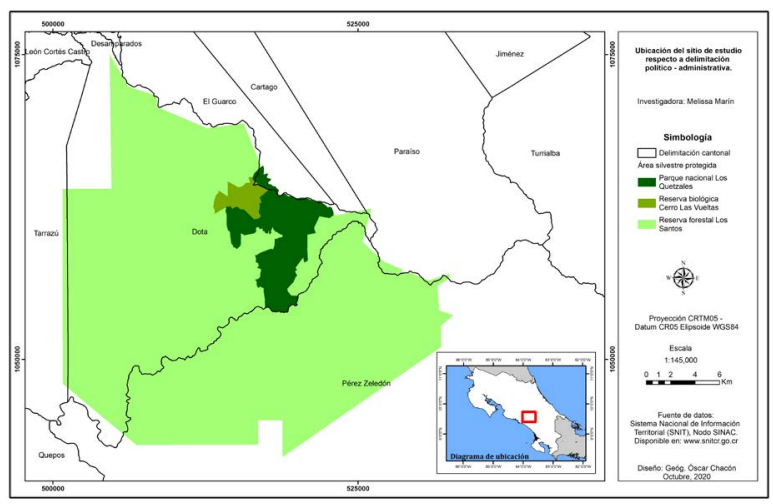

Figura 1. Ubicación del área de estudio.

Fuente: Propia de la investigación con datos obtenidos del Sistema Nacional de Información Territorial.

\section{Elementos de análisis}

Los elementos de análisis son grupos organizados formales e informales (nodos) con las siguientes características: a) sus objetivos son el desarrollo, la conservación ambiental, el manejo y aprovechamiento de los recursos naturales (que incluye turismo y recursos hídricos), la educación ambiental, la investigación, o la producción, b) su área de influencia son las áreas silvestres protegidas antes mencionadas, y c) sus miembros se ubican en estas mismas áreas.

Siguiendo lo planteado por Molina (2001) sobre la importancia de trabajar con redes completas y según el enfoque nominalista de Verd y Olivé (1999), se identificaron los límites del universo de actores mediante el método no probabilístico de muestreo en cadena. 
El total de grupos organizados analizados fue de 40 en 2009-2010 y 40 en 2019-2020.

\section{Variables atributivas}

Las variables atributivas fueron: a) tipología general: institución de gobierno, organización social, sector privado, grupos multisectoriales, otro; b) tipología específica: institución de gobierno, organizaciones productivas, ASADAS, Asociación de Desarrollo Integral (ADI), Asociaciones de Desarrollo (AD), Organizaciones No Gubernamentales (ONG), sector privado, grupos multisectoriales, cooperativas, centros de investigación, organizaciones de desarrollo, otro; c) área geográfica de influencia: nivel micro (comunidades o áreas protegidas) y nivel meso (cantón de Dota); d) objetivo principal: desarrollo, producción, aprovechamiento de recursos naturales, conservación ambiental, manejo de recursos naturales, investigación y educación ambiental.

\section{Unidades de análisis e indicadores}

Las unidades de análisis son las interacciones (vínculos) entre los grupos organizados.

En el nivel global (red), los indicadores analizados fueron: a) densidad: medida de cohesión de la red, son las conexiones potenciales en función a las reales y que reflejan la eficacia ante cambios; b) distancia geodésica: distancia media más corta entre dos grupos; d) número de interacciones: indica cohesión de la red; c) diámetro: distancia geodésica más larga de la red, es una medida de cohesión; e) centralización global (Freeman, 1979): medida de dominancia de los grupos, y la posibilidad de que si estos son removidos la red se fragmente; g) centralización entrada y salida: en el primer caso, indica el prestigio y poder de la red y en segundo caso, indica su actividad social.

En el nivel posicional (por cada grupo organizado) los indicadores fueron: a) grado de centralidad: conexiones directas; b) vínculos de entrada: identifica los más prestigiosos y de referencia para los demás, que suelen ser depositarios de información; c) vínculos de salida: indica actividad social y su capacidad de difundir información; d) grado de cercanía: facilidad que tiene para acceder a todos los demás; e) grado de intermediación: control que tiene para facilitar u obstaculizar el flujo de información en la red; f) centralidad beta (Bonacich, 1987): mide el poder en función de cuántas conexiones tienen los actores en relación con él; g) puntos de corte: puntos donde se interrumpe la conexión entre subgrupos.

\section{Tipos de relaciones}

Se analizan las características de las relaciones de comunicación ya sean informales (para solicitud de información o asesorías) o formales (para coordinación de acciones relacionadas con medio ambiente).

\section{Instrumentos}

La recopilación de información se realizó mediante la observación participante, información secundaria y la aplicación de entrevistas semiestructuradas en diferentes espacios temporales. Estas entrevistas se implementaron en su gran mayoría de forma presencial, pero algunas de ellas se realizaron puntualmente vía telefónica (debido a la pandemia del COVID-19).

Por medio de los programas Microsoft Excel y UCINET VI, se almacenaron y manejaron los datos utilizando el formato matriz con el mismo número de filas $\mathrm{y}$ columnas. 
La traducción gráfica se hizo mediante redes sociocéntricas de vínculos dirigidos y matrices asimétricas y dicotómicas donde 1 fue presencia y 0 ausencia de relación. Los datos se visualizaron y analizaron con la teoría de grafos y matrices relacionales y de adyacencia utilizando NetDraw y UCINET VI (Borgatti, Everett y Freeman, 2002).

Con los mapas de calor se muestra el agrupamiento geográfico de las áreas de actuación de los nodos según los diferentes indicadores.

\section{Análisis y resultados}

\section{Representatividad de los grupos organizados en las redes según tipología}

En el primer análisis en 2009, la red organizacional se conformó por 40 nodos, de los cuales $65 \%$ fueron organizaciones sociales (9 AD y ADI, 6 organizaciones de producción, 4 ASADAS, 4 cooperativas, 2 ONG, 1 organización de desarrollo); 15\% instituciones de gobierno; $10 \%$ grupos multisectoriales; 7,5\% sector privado; y $2,5 \%$ otro.

En el segundo análisis en 2020, la red estuvo integrado igualmente por 40 nodos, de los que 62,5\% fueron organizaciones sociales (9 AD y ADI, 2 organizaciones de producción, 3 ASADAS, 1 ONG, 5 son cooperativas, 1 organización de desarrollo, y 4 son otros); $22,5 \%$ instituciones de gobierno; $10 \%$ sector privado; $2,5 \%$ grupos multisectoriales; y $2,5 \%$ otro.

Los mayores cambios se dieron en las instituciones de gobierno (aumentaron un 7,5\%) y del sector privado (que aumentó en un 2,5\%). Por el contrario, las organizaciones sociales y los grupos multisectoriales disminuyeron su presencia un $2,5 \%$ y $7,5 \%$ respectivamente. La categoría de 'otros' se mantuvo con el mismo representante.

Eficiencia de las redes en la distribución de oportunidades, recursos e información

Considerando lo expuesto por Aguilar, Martínez y Aguilar (2017), de que en las redes grandes la densidad tiene valores comúnmente bajos, debido a que la cantidad de vínculos posibles es proporcional al tamaño de la red (p. 78), cabría esperar valores relativamente bajos pero similares en las densidades de las redes del 2009 y 2020, puesto que la cantidad de grupos se mantuvo idéntico. Sin embargo, los vínculos existentes frente a los posibles se redujeron en un $45 \%$, pasando de 296 a 163. La densidad de la red pasó del $19 \%$ a $10,4 \%$ y la cohesión disminuyó un 45\% (ver gráficas 1 y 2).

Lo anterior implica que comparativamente hubo una disminución importante en la capacidad de la red de mantenerse unida ante cualquier eventualidad, en establecer y mantener vínculos, y en la velocidad de difusión de información y oportunidades. Todo lo anterior afecta el acceso a los recursos, siendo estos más eficientemente distribuidos en 2009 . 


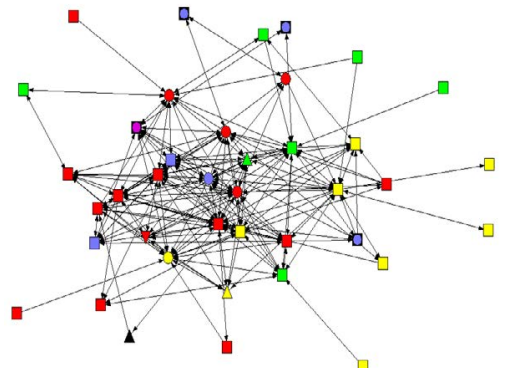

Gráfica 1. Red social en 2009 en la Zona de Los Santos, Costa Rica.

Fuente: Propia de la investigación.

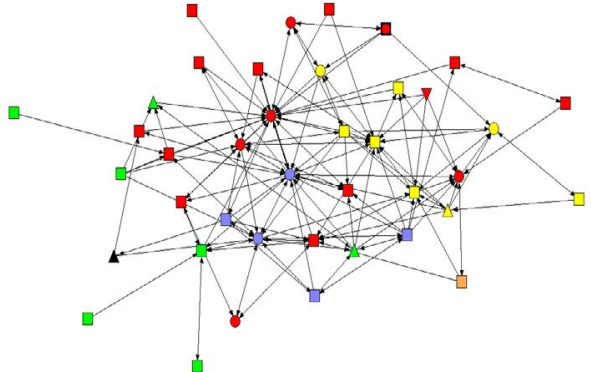

Gráfica 2. Red social en 2020 en la Zona de Los Santos, Costa Rica.

Fuente: Propia de la investigación.

Nota: Los códigos por objetivo del grupo: desarrollo (rojo), producción (amarillo) aprovechamiento de recursos naturales (verde), conservación ambiental (azul), manejo de recursos naturales (morado), investigación (negro) y educación ambiental (anaranjado). Por tipología del grupo: instituciones de gobierno (círculo), organizaciones sociales (cuadrado), sector privado (triángulo hacia arriba), grupos multisectoriales (círculo dentro de cuadrado) y otro (triángulo hacia abajo).

\section{Control y acceso a la información y oportunidades en la red}

Los cambios en el índice de centralidad de la red y sus grados de entrada, tal y como mencionan Aguilar et al. (2017), sugieren la dominancia de uno o varios grupos como receptores de información pero, a la vez, una disminución considerable en la transmisión de esa información. Es decir, el control de la información y oportunidades está más centralizado en 2020 por la presencia de grupos con mayor prestigio social.

Lo anterior se corrobora también con el índice de centralización, que para el 2009 fue de $6,97 \%$, mientras que para 2020 de $19,94 \%$. Los grados de entrada pasaron de $38,4 \%$ en 2009 a $44,5 \%$ en 2020 , y los grados de salida pasaron de $33,1 \%$ en 2009 a $18,2 \%$ en 2020.

Igualmente, considerando la mayor distancia necesaria de desplazamiento para que cualquier grupo acceda a otro, de 2009 a 2020 aumentó un 50\% el esfuerzo que debía invertir cada uno para este fin (el diámetro de la red pasó de 4 a 6). La distancia media más corta entre grupos organizados aumentó de 2,05 a 2,7 entre ambos años.

El índice de compactación de la red para el 2009 fue de 0,484 y de 0,382 en 2020 , lo que igualmente sugiere un aumento en la desvinculación de los nodos durante ese período. La mayor insuficiencia de enlaces en 2020 con respecto a 2009 muestra el surgimiento de grupos desconectados y con mayor dificultad de acceder a la información y oportunidades.

Por otro lado, la influencia potencial de los grupos organizados fue un 7,4\% más en 2020, y su índice de intermediación aumentó un $62 \%$. Este poder de intermediación sobre la información está mayormente centralizado en las instituciones de gobierno (ver tabla 1 ). 


\begin{tabular}{|c|c|c|c|c|c|c|c|c|}
\hline \multirow{2}{*}{ TIPOLOGÍA } & \multicolumn{2}{|c|}{ n-outdegree } & \multicolumn{2}{|c|}{$n$-indegree } & \multicolumn{2}{|c|}{ n-betweenness } & \multicolumn{2}{|c|}{ n-Bonachic power } \\
\hline & 2009 & 2020 & 2009 & 2020 & 2009 & 2020 & 2009 & 2020 \\
\hline Gobierno & 1,821 & 1,231 & 1,976 & 2,052 & 29,696 & 96,591 & 6,411 & 12,376 \\
\hline Organización social & 4,489 & 2,156 & 3,563 & 1,846 & 56,115 & 45,255 & 17,055 & 15,499 \\
\hline Sector privado & 0,513 & 0,435 & 0,513 & 0,257 & 1,564 & 5,160 & 1,414 & 1,000 \\
\hline Multisectorial & 0,487 & 0,103 & 0,487 & 0,026 & 2,992 & 0,328 & 1,230 & 0,141 \\
\hline Otro & 0,256 & 0,103 & 0,179 & 0,000 & 0,413 & 0,000 & 0,890 & 0,000 \\
\hline
\end{tabular}

Tabla 1. Indicadores posicionales comparativo años 2009 y 2020. Fuente: Propia de la investigación.

Los puntos de corte de la red también disminuyeron en cantidad y diversidad. Para 2009 son dos instituciones de gobierno, un grupo de mujeres, una cooperativa y una asociación de productores regional (estas últimas desaparecieron para el 2020). La desaparición de estos grupos fraccionaría la red en siete bloques.

Para 2020 los puntos de corte son una institución de gobierno, una asociación de desarrollo y una ASADA. Su desaparición fraccionaría la red en cinco bloques.

\section{Influencia y poder de los diferentes grupos organizados según tipología}

En las dos redes analizadas las organizaciones sociales representaron más del $60 \%$ de los nodos. En 2009, estas tenían la mayor distribución de capacidades y poder, y las mayores centralidades. Dos cooperativas y un grupo regional ocupaban los tres primeros puestos en intermediación de toda la red, lo que refleja la importancia de los colectivos regionales como mediadores. En 2020, el poder dentro de este subgrupo pasó a estar más centralizado.

Las instituciones de gobierno, aunque tienen en la totalidad menos poder que las organizaciones sociales, sí tienen más de lo que se espera considerando la cantidad de nodos que los representa en la red. La única institución dedicada a conservación ambiental se coloca como tercera en importancia en toda la red en cuanto al poder por la calidad de sus contactos.

Para el 2020, a pesar de que las instituciones de gobierno tienen $64 \%$ menos nodos que las organizaciones sociales, tienen los mayores índices indegree e intermediación. Esto quizá tenga relación con la incorporación de 3 instituciones del Estado, una de ellas, la oficina para la Reserva Biológica Cerro Vueltas y el Parque Nacional Los Quetzales, que abrió en 2007 y que se ha visto mejor posicionada desde su creación hasta la actualidad; con la elaboración de los Planes de Manejo de la Reserva Biológica Cerro Vueltas en 2015 y del Parque Nacional Los Quetzales en 2017; y con la desaparición de las 4 plataformas multisectoriales ambientales, quedando ausentes en la red de 2020. Este espacio pudo haber sido ocupado por las instituciones de gobierno enfocadas en este tema.

La diversificación en cuanto a los grupos que ejercen mayor actividad social o capacidad de emitir y recibir información se redujo y se centralizó. En el 2009 esta función estaba centralizada en dos cooperativas, una agrupación regional de productores orgánicos, una institución de gobierno y dos asociaciones de desarrollo. Los que contaban con mayor prestigio fueron tres instituciones de gobierno y dos cooperativas. En el 2020, la actividad social se centralizó en tres instituciones de gobierno, 
una cooperativa y una organización social de enfoque regional, y el prestigio, en tres instituciones de gobierno y una cooperativa.

Llama la atención en 2020 la ausencia de asociaciones de desarrollo, entre los principales dinamizadores de la red, a diferencia de 2009 donde estaban mucho mejor posicionadas. Con respecto a 2009 , estas redujeron un $65 \%$ su actividad social, un $62 \%$ su prestigio, y un $20 \%$ su capacidad mediadora. Igualmente, la calidad de sus contactos disminuyó un 50\% aproximadamente.

A pesar de la importancia del recurso hídrico en las tres áreas silvestres protegidas, la incidencia y el poder de las ASADAS es de las más bajas. En el 2009 el $100 \%$ de ellas tuvieron -entre las organizaciones sociales- los índices más bajos en la distribución de información, intermediación y calidad de sus contactos. En 2020, tienen mucho más control del flujo de información porque su intermediación mejora sustancialmente (pasa de 0,281 a 7,720); esto aumentó su capacidad mediadora y sus posibilidades para establecer alianzas. Pese a ello, aún continúan dentro de los grupos con las centralidades más bajas.

Lo anterior sugiere que, si bien el recurso hídrico es clave para el desarrollo y que es un tema que puede enlazar a gran cantidad de actores, aún los grupos para la gestión local de este recurso se perciben así mismos y son percibidos solo como administradores, y no como parte de una estructura de relaciones de conservación y desarrollo en la región.

Los grupos de producción orgánica tuvieron en 2009 los niveles más bajos de centralidad, capacidad para mantener vínculos y poder local. Dos de estos cuatro grupos no tenían vínculos de salida y solo uno de entrada con la única organización regional de producción. Esta sí tenía altas capacidades de establecer vínculos y recibirlos, y su intermediación fue la tercera en importancia en la red; pero al no tener conexiones con grupos bien conectados, su poder se redujo.

Todos los grupos de producción orgánica local y regional desaparecieron de la red en 2020. Esta actividad continúa desarrollándose en el área, pero de forma privada y desvinculada de la red organizacional.

Las cooperativas redujeron su actividad social un 50\% en 2020; sin embargo, dentro de las organizaciones sociales, son de los que tienen el mayor poder y centralidad.

El sector privado no está suficientemente representado en la red (solo tres nodos en 2009 y cuatro en 2020). Su capacidad de emitir y recibir información se reduce para el 2020, aunque su intermediación se vio aumentada. En ambos años, su poder está concentrado en un grupo que representa a propietarios privados y de influencia regional.

En 2009, las plataformas multisectoriales eran cuatro (todas con objetivos sobre medio ambiente). Estas son estrategias de gobernanza que, a falta de un COLAC, se crean para impulsar las mesas de diálogo. Sin embargo, en 2009, tres de ellas tenían capacidades bajas de emitir información, lo que redujo considerablemente su intermediación (función clave de estos colectivos). La única plataforma especializada en recursos hídricos sí tenía niveles de indegree y outdegree altos, al igual que la calidad de los contactos y su centralidad, por lo que se ubicó entre los grupos con más poder.

Sin embargo, debido a que en el área de estudio estas plataformas son creadas en respuesta a metas concretas en el tiempo, para 2020 todas desaparecieron una vez alcanzado el objetivo. Surge una plataforma enfocada en desarrollo, pero con muy bajos índices de poder. 
La categoría "otro" está representada por un solo grupo organizado. En 2009 su valor en outdegree e indegree superaba ligeramente la media en la red, pero en 2020 fue cero. Esto quizá porque en 2020 sus funciones fueron muy específicas y su rango de acción se encuentra mayoritariamente fuera del área de estudio.

\section{Predominancia de objetivos gene- rales (temas) en las redes}

En las redes analizadas, la centralidad estaba dominada por el tema de desarrollo, que además es el único que aumenta en presencia para el 2020; y, por el tema de conservación, a pesar de que ocupa el cuarto puesto en cantidad de grupos especializados en este tema (ver gráficas 1, 2 y 3 ).

En términos generales, con respecto al 2009 , todos los temas disminuyeron su poder en 2020 entre el 40 y 70\%, a excepción del tema de conservación que fue el que menos variación tuvo (disminuyó en $26 \%$ su poder) y el de manejo de recursos naturales que desapareció de la red. Surge la educación ambiental como una nueva prioridad.
El desarrollo posee los valores más altos en casi todos los indicadores a pesar de que para el 2020 en este se tema disminuyó un $57 \%$ la actividad social, un $45 \%$ el prestigio, un $45 \%$ la calidad de los vínculos y su capacidad mediadora aumentó un 17\%. Los intereses en desarrollo siguen siendo claves, pero su capacidad de dinamización no es tan alta como antes. Esto posiblemente porque menos grupos con más estabilidad económica y menos actividad social controlan el tema, lo que reduce su alcance.

La producción disminuyó en más del $30 \%$ en todos los indicadores, aunque aumenta un $7,5 \%$ en intermediación. Esta centralidad está concentrada en instituciones y grupos regionales que representan colectivos, siendo los grupos locales los de menor actividad social y prestigio. Esto pudo haber influenciado que la producción orgánica desapareciera de la red para el 2020.

El aprovechamiento de los recursos se mantiene con la misma cantidad de grupos, principalmente especializados en turismo y recurso hídrico. Sin embargo, en la red de 2020 este tema se desplaza hacia la periferia (ver gráficos 1 y 2) y se es más vulnerable

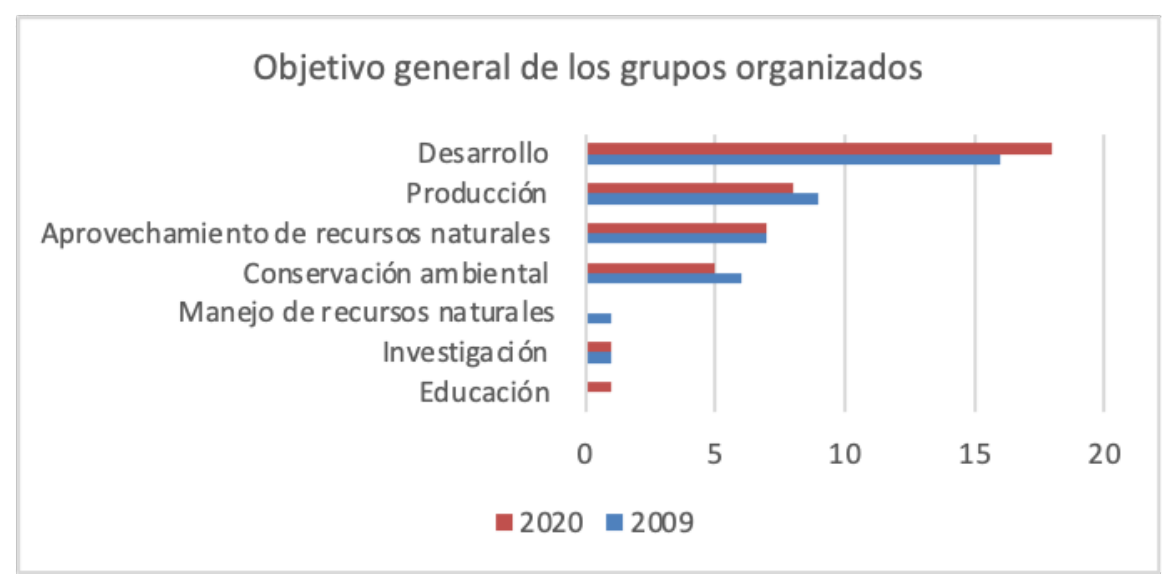

Gráfica 3. Cantidad de grupos organizados especializados en un objetivo general en 2009 y 2020. Fuente: Propia de la investigación. 
porque pasa de uno a tres nodos con un solo vínculo; esto hace que su poder disminuya un $70 \%$.

La conservación ambiental es la que más ha tenido cambios en su composición, pero a la vez el más resiliente. Con respecto al 2009, en 2020 desaparecen las tres organizaciones multisectoriales y las dos $\mathrm{ONG}$ dedicadas a este tema, permanece solo la institución de gobierno y surgen dos grupos más. Sin embargo, la centralidad que le da la institución de gobierno hace que la conservación se mantenga como prioridad en el área. En 2020 este tema ocupó el segundo y tercer puesto en actividad social, intermediación y poder.

La investigación es la que tuvo menor poder en ambas redes. Si se considera la poca representación de este sector y su poca capacidad de incidencia en una zona que incluye tres áreas silvestres protegidas, resulta preocupante la capacidad de la red de acceder a información científica para la toma de decisiones sobre medio ambiente.

El manejo de los recursos naturales también se encuentra subrepresentado en la red, con solo un grupo específico sobre recurso hídrico. Su actividad social es un poco más alta de la esperada, así como su prestigio. En este tema se priorizan las relaciones con plataformas multisectoriales o instituciones de gobierno, y con pocos vínculos con las ASADAS. El manejo de recursos naturales desapareció como tema en la red de 2020.

La educación ambiental surge en el 2020 como un nuevo objetivo general y representado por un grupo organizado. Por ahora tiene poco poder porque actúa en el área adyacente del área de estudio, y recién inicia acciones en la Reserva Forestal Los Santos. Sin embargo, la mayoría de los grupos sí realiza actividades sobre educación ambiental (para concienciación y aumento de capacidades), lo cual muestra que quizá este tema está siendo percibido como una herramienta para alcanzar determinados objetivos generales.

\section{Áreas de influencia}

Las áreas geográficas de influencia de los indicadores de centralidad se analizan desde el punto de vista micro (comunidades $\mathrm{o}$ áreas silvestres protegidas) y meso (a nivel regional, es decir del cantón de Dota). Los seis principales resultados fueron:

1) De 2009 a 2020 los grupos que inciden a nivel micro disminuyeron en cantidad, ya que pasaron de 57,5\% a 47,5\% (4 nodos menos), mientras que los que inciden a nivel meso aumentaron, pasando de $42,5 \%$ a 52,5\% (4 nodos más). Muchas de las comunidades de estudio pasaron a ser dependientes de grupos regionales.

2) En ambas redes, la zona donde confluyen las tres áreas silvestres protegidas fue la que tuvo mayor influencia de los indicadores de centralidad (ver figuras 2 y 3 ).

3) En 2009, esta posición correspondió a la cuenca del río Savegre y el elemento diferenciador fue la influencia de asociaciones locales y la de una cooperativa que surgió como resultado de la creación del Parque Nacional Los Quetzales. Debido a esto, Providencia y San Gerardo de Dota fueron las comunidades mejor posicionadas en actividad social y búsqueda de alianzas (por encima del mismo parque nacional con el cual limitan). Sin embargo, la desaparición de esta cooperativa pudo haber causado que en 2020 ambas hayan pasado a ser dos de las que 
recibieron menos influencia de la red (ver figura 3). Actualmente, estas dos comunidades poseen altos índices de actividad social principalmente por la promoción del ecoturismo, pero se da a nivel privado, con poca vinculación con las redes de grupos organizados. Esto podría disminuir su capacidad de resiliencia, por ejemplo, frente a cambios en el mercado. En las entrevistas realizadas en 2019 los grupos organizados en Providencia y San Gerardo no priorizaron acciones en conservación ambiental debido a que se considera que en este tema existe un nivel óptimo; que la conservación se percibe como de responsabilidad gubernamental; y que las instituciones de gobierno encargadas de la gestión ambiental no establecen vínculos ni canales de comunicación eficientes con estas comunidades.

4) Para el 2020 surge el área de la carretera interamericana o Cerro de la Muerte como la de mayor influencia de los indicadores, especialmente en conservación ambiental y en el aprovechamiento de los recursos naturales, sustituyendo, en este sentido, a la

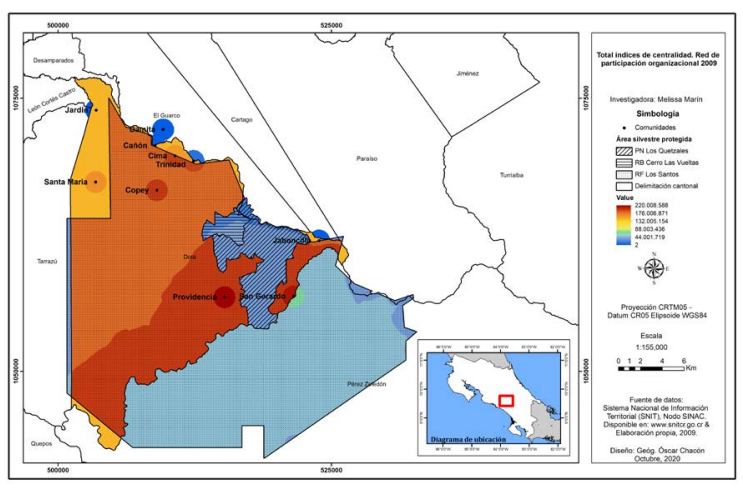

Figura 2. Áreas geográficas de influencia de los índices de centralidad en 2009. Fuente: Propia de la investigación. cuenca del río Savegre. En esta área se concentran las comunidades con las mayores centralidades en el aprovechamiento del recurso hídrico asociado a la Reserva Biológica Cerro Vueltas.

5) La Reserva Forestal Los Santos fue el área que ocupó el segundo lugar en influencia sobre actividad social y prestigio y en donde se tienen mejores conexiones. Esto no es de sorprender, puesto que su categoría de manejo permite un aprovechamiento de los recursos más amplio e inclusive poblados viviendo dentro de sus límites. Esto hace que se dé allí una mayor interacción social con respecto a las otras áreas silvestres protegidas. Las organizaciones que inciden exclusivamente en esta área se enfocan en la conservación y en mucho menor medida en la producción.

6) Las dos redes analizadas no extendieron vínculos hacia el sureste de la reserva forestal, que corresponde al cantón de Pérez Zeledón y donde no existen otras áreas silvestres protegidas. Esto posiblemente obedezca a razones geográficas o históricas.



Figura 3. Áreas geográficas de influencia de los índices de centralidad en 2020.

Fuente: Propia de la investigación. 


\section{Conclusiones}

La participación en Costa Rica ha sido un elemento clave en la gestión de territorio. La presencia de redes con más de $60 \%$ de organizaciones sociales muestra la importancia que le da la sociedad a la gestión y la toma de decisiones participativa.

Las redes de participación organizacional analizadas centralizan su interés alrededor de los dos grandes temas: la conservación ambiental y el desarrollo. En medio de ellos están el aprovechamiento y manejo de los recursos naturales, la producción, la investigación y la educación ambiental, que le dan funcionalidad al desarrollo sostenible pero que en la práctica aparecen, desaparecen, reducen su poder o lo aumentan ante los cambios estructurales en las redes. Esta fluctuación hace que su influencia disminuya, lo que, a nivel práctico, debilita los puntos de encuentro entre la conservación y el desarrollo.

Las áreas silvestres protegidas son claves como elementos dinamizadores de las redes de participación de grupos organizados, ya que las acciones de estos grupos son más intensas en las áreas donde confluyen dos o más áreas protegidas. En este caso, no se identifican subredes específicas para cada área, sino que la participación se construye como un solo bloque con influencias variables en determinadas áreas geográficas.

Áreas silvestres protegidas de categorías más estrictas están más enfocadas en la conservación y reciben una menor incidencia de las redes de participación organizacional. Áreas con categorías menos estrictas tienden a priorizar el desarrollo frente a los demás temas y tienen redes más dinámicas, más diversas y con más juegos de poder.

En el Parque Nacional Los Quetzales o la Reserva Biológica Cerro Vueltas, las posibilidades de aprovechar los recursos están más regularizadas mediante su plan de manejo y esto, junto a un menor acercamiento multisectorial, produce que los grupos que actúan aquí tengan poca presencia, estén desvinculados de la red, o estén más enlazados con el sector privado.

Las tres áreas silvestres protegidas limitan entre sí, por lo que la Reserva Forestal Los Santos posiblemente sea un amortiguador que disminuye la presión sobre las otras dos áreas.

Hay que considerar que la Reserva Forestal Los Santos no posee plan de manejo, por lo que el uso de sus recursos está sujeto a las regulaciones del Patrimonio Natural del Estado. Esto produce una mayor presión extractiva, y una mayor intervención de los grupos $\mathrm{y}$, con ellos, mayor diversidad de intereses y conflictos. Lo anterior es lógico considerando que es en este espacio donde se consolidan las oportunidades de desarrollo.

La actividad social de los grupos está ligada con la capacidad de la red de distribuir y aprovechar de forma adecuada las oportunidades y la información. Redes sociales más centralizadas y que priorizan la recepción frente a la emisión de información se vuelven menos diversificadas en sus puntos de vista y enfoques de trabajo, más controladoras y vulnerables. Esto afecta la calidad de los procesos participativos, afecta el capital social y su capacidad de resiliencia ante cualquier eventualidad.

A mayor diversificación de actores en las redes, mayor eficiencia y sostenibilidad en la distribución de información y oportunidades. En este sentido, la red de 2009 muestra una mayor eficiencia que la del 2020.

La ausencia de plataformas de coordinación y una disminución en la centralidad de las asociaciones de desarrollo parecen 
ser los elementos que alteran mayormente la funcionalidad de las redes sobre las áreas silvestres protegidas. Lo anterior produce una mayor centralización del poder por parte de los grupos regionales, además de mayor control en la definición de los temas prioritarios. Esto disminuye la eficiencia de la red en la distribución de oportunidades a nivel comunitario y hace que la innovación (por ejemplo, en sistemas productivos orgánicos) se vuelva más difícil a lo interno de estas redes.

La resiliencia del tema de conservación está vinculada por el poder que da la presencia de la institución de gobierno que gestiona las tres áreas protegidas. Esto podría aumentar su eficacia en el cumplimiento de los objetivos de conservación, pero en ausencia de plataformas multisectoriales, este poder disminuye, así como su ámbito de influencia.

Atendiendo a la hipótesis planteada, se concluye que:

a) Desde la dimensión relacional (Sabatini, 2009), las formas de distribución de poder, es decir, las características estructurales de las redes sociales que se crean y evolucionan alrededor de las áreas silvestres protegidas sí son influenciadas por las posibilidades de aprovechar los recursos naturales que se protegen dentro de estas áreas. En este sentido, sí existe relación directa entre las características estructurales de las redes sociales y las categorías de manejo de las áreas silvestres protegidas, en la medida que estas pueden ofrecer más elementos de intercambio, que son los que dinamizan las redes. Estos elementos dinamizadores podrían compararse con satisfactores (según el enfoque de Desarrollo a Escala
Humana de Neef et al., 1986) y que tiene su base en función de las necesidades humanas universales.

b) Desde la dimensión funcional de las redes sociales (Häuberer, 2011) -es decir, la eficiencia y el impacto que estas tengan sobre el desarrollo- no existe vinculación directa con la categoría de manejo del área protegida, pero sí con las capacidades locales y el poder de las redes para hacer accesibles los satisfactores. Aquí influyen aspectos culturales, históricos, sociales, políticos, entre otros, independientemente de la categoría de manejo del área protegida.

Finalmente, las áreas silvestres protegidas son un componente más del desarrollo ya que, al posicionar la variable ambiental en la planificación territorial, aseguran a perpetuidad la generación de servicios ecosistémicos para satisfacer las necesidades humanas. Sin embargo, es en las redes sociales alrededor de estas áreas donde estos servicios pueden transformarse o no en oportunidades para el desarrollo sostenible. Por ello, es imprescindible considerar la efectividad de estos espacios de interacción, como un elemento clave de sostenibilidad en la planificación territorial dentro y fuera de las áreas silvestres protegidas.

\section{Agradecimientos}

Se agradece al programa de Doctorado de Ciencias Naturales para el Desarrollo; y a las instituciones, grupos organizados y comunidades de la zona de Los Santos. 


\section{Conflicto de intereses}

La autora declara no tener algún conflicto de interés.

\section{Declaración de la contribución de los autores}

Se afirma que se leyó y aprobó la versión final de este artículo. El porcentaje de contribución en la conceptualización, preparación y corrección fue: M.M.C. 100 \%.

\section{Declaración de disponibilidad de los datos}

Los datos que respaldan los resultados de este estudio serán puestos a disposición por la autora correspondiente M.M.C., previa solicitud razonable.

\section{Referencias}

Aguilar, N., Martínez, E. y Aguilar, J. (2017). Análisis de redes sociales: Conceptos clave y cálculo de indicadores. Universidad Autónoma Chapingo (UACh), México. Serie: Metodologías y herramientas para la investigación. Volumen 5.

Ávila, J. (2012). Redes sociales y análisis de redes: Aplicaciones en el contexto comunitario y virtual. Corporación Universitaria Reformada.

Benavides, S. (2020). El aporte del turismo a la economía costarricense: Más de una década después. Economía y Sociedad, 25(57), 1-29. 10.15359/eys.25-57.1

Bodin, Ö. and Crona, B. (2009). The role of social networks in natural resource governance: What relational patterns make a difference? Global Environmental Change, 19(3), 366-374. doi. org/10.1016/j.gloenvcha.2009.05.002

Bonacich, P., (1987). Power and Centrality: A Family of Measures. American Journal of Sociology, 92(5), 1170-1182.

Borgatti, S., Everett, M. and Freeman, L. (2002). Ucinet for Windows: Software for Social Network Analysis. Analytic Technologies.

Coleman, J. (1990). Foundation of Social Theory. The Belknap Press of Harvard University Press.

Diani, M. and McAdam, C. (2003). Social Movements and Networks, Contentious Actions, and Social Networks: 'From Metaphor to Substance'? Oxford University Press. 10.1093/0199251789.003.0001

Dudley, N. (Ed.) (2008). Directrices para la aplicación de las categorías de gestión de áreas protegidas. UICN. https://portals.iucn.org/library/efiles/documents/PAPS-016-Es.pdf

Freeman, L. (1979). Centrality in social networks: conceptual clarification. Social Networks, 1(3), 215-239. doi: 10.1016/0378-8733(78)90021-7

Gentry, A. (1982). Neotropical floristic diversity: phytogeographycal connections between Central and South America, Pleistocene climatic fluctuations, or an accident of the orogeny. Annals of the Missouri Botanical Garden, 69(3), 557-593. doi: 10.2307/2399084

Hanneman, R. (2000). Introducción a los métodos de análisis de redes sociales. Departamento de 
Sociología, Estados Unidos: Universidad de California.

Häuberer, J. (2011). Social capital theory. Towards a methodological foundation. Germany: Springer Fachmedien.

Kappelle, M. and Brown, A. (2001). Bosques nublados del neotrópico. INBio.

Mardones, G. (2017). Análisis de redes sociales para la gobernanza de un área protegida y su zona de amortiguación en el bosque templado del sur de Chile. Revista Hispana para el Análisis de Redes Sociales, 28(1), 61-72.

Maya, I., Florido del Corral, D. and Sáenz, J. (2017). Redes de consejo y apoyo social de las embarcaciones autorizadas a pescar en la reserva de la desembocadura del Guadalquivir: Tres estrategias de pesca desde el puerto de Chipiona. Revista Hispana para el Análisis de Redes Sociales, 28(1), 32-46. 10.5565/rev/ redes.651

Molina, J. (2001). El análisis de redes sociales, una introducción. Barcelona.

Neef, M., Elizalde, A. and Hopenhayn, M. (1986). Desarrollo a escala humana: Una opción para el futuro. Development Dialogue, número especial. CEPAUR, Fundación Dag Hammarskjold.

Palacio, D. (2017). El lugar-red y la acción ambiental. Pistas para una gobernanza reflexiva y situada. Revista Hispana para el Análisis de Redes Sociales, 28(1), 73-91. org/10.5565/ rev/redes.648

Programa Estado de la Nación en Desarrollo Humano Sostenible-Costa Rica (2014). Estado de la Ciencia, la Tecnología y la Innovación. PEN.
Sabatini, F. (2009). Social capital as social networks: A new framework for measurement and an empirical analysis of its determinants and consequences. The Journal of Socio-Economics, 38(3), 429-442. 10.1016/j.socec.2008.06.001

Samper, M. (2004). Redes sociales y comunicación entre experimentadores campesinos en Puriscal, Costa Rica. Revista Ciencias Sociales, 4(106), 143-163.

Sistema Nacional de Áreas de Conservación-SINAC. (2015). Plan de Manejo de la Reserva Biológica Cerro Vueltas. Área de Conservación Pacífico Central (ACOPAC). Costa Rica

Sistema Nacional de Áreas de Conservación-SINAC. (2017). Plan General de Manejo del Parque Nacional Los Quetzales. Área de Conservación Pacífico Central (ACOPAC). Costa Rica.

Sistema Nacional de Áreas de Conservación-SINAC. (2020). Control de áreas silvestres protegidas por categoría de manejo. http://www. sinac.go.cr/ES/asp

Verd, J. y Olivé, J. (1999). Muestreo y recogida de datos en el análisis de redes sociales. $Q \ddot{U} E S$ TIIÓ, 23(3), 507-524. Universitat Autònoma de Barcelona.

Wasserman, S. and Faust, K. (1994). Social Network Analysis: Methods and Application. Structural analysis in the social sciences. Cambridge University Press. United Kingdom.

World Travel and Tourism Council-WTTC. (2019). The economic impact of global wildlife tourism. United Kingdom.

\section{(c) (i) $(\mathrm{BY})$}

Redes sociales en áreas silvestres protegidas de Costa Rica (Melissa Marín Cabrera) Uniciencia is protected by Attribution-NonCommercial-NoDerivs 3.0 Unported (CC BY-NC-ND 3.0) 